\title{
Using structural equation modeling to investigate change and response shift in patient-reported outcomes: practical considerations and recommendations
}

\author{
M. G. E. Verdam ${ }^{1,2}$ (D) F. J. Oort ${ }^{3}$ (1) $\cdot$ M. A. G. Sprangers ${ }^{2}$
}

Accepted: 21 December 2020 / Published online: 7 February 2021

(c) The Author(s) 2021

\begin{abstract}
Background Patient-reported outcomes (PROs) are of increasing importance for health-care evaluations. However, the interpretation of change in PROs may be obfuscated due to changes in the meaning of the self-evaluation, i.e., response shift. Structural equation modeling (SEM) is the most widely used statistical approach for the investigation of response shift. Yet, non-technical descriptions of SEM for response shift investigation are lacking. Moreover, application of SEM is not straightforward and requires sequential decision-making practices that have not received much attention in the literature.

Aims To stimulate appropriate applications and interpretations of SEM for the investigation of response shift, the current paper aims to (1) provide an accessible description of the SEM operationalizations of change that are relevant for response shift investigation; (2) discuss practical considerations in applying SEM; and (3) provide guidelines and recommendations for researchers who want to use SEM for the investigation and interpretation of change and response shift in PROs.

Conclusion Appropriate applications and interpretations of SEM for the detection of response shift will help to improve our understanding of response shift phenomena and thus change in PROs. Better understanding of patients' perceived health trajectories will ultimately help to adopt more effective treatments and thus enhance patients' wellbeing.
\end{abstract}

Keywords Structural equation modeling (SEM) · Patient-reported outcomes (PROs) · Health-related quality of life $(\mathrm{HRQL}) \cdot$ Change $\cdot$ Response shift

\section{Introduction}

Patient-reported outcomes (PROs) are increasingly recognized as a critical endpoint in health care and medicine, and routine assessment of PROs is becoming standard part of

Supplementary Information The online version contains supplementary material available at https://doi.org/10.1007/s1113 6-020-02742-9.

M. G. E. Verdam

m.g.e.verdam@fsw.leidenuniv.nl

1 Department of Methodology and Statistics, Institute of Psychology, Leiden University, P.O. Box 9555, 2300 RB Leiden, The Netherlands

2 Department of Medical Psychology, Amsterdam University Medical Centre, Amsterdam Public Health Research Institute, Amsterdam, The Netherlands

3 Research Institute Child Development and Education, University of Amsterdam, Amsterdam, The Netherlands clinical practice [1]. The importance of measuring PROs, such as health-related quality of life (HRQL), is especially salient in view of aging societies and more powerful healthcare interventions, which have led to an increasing number of people living with chronic disease [2]. That is, the ultimate purpose of health-care interventions may often not be prolonged survival but maintenance or optimization of patients' quality of life [3].

Evaluating the impact of disease and treatment on patients' perceived health trajectories requires longitudinal assessment. However, interpretation of change in PROs is complicated by the fact that the meaning of respondents' self-evaluations may change too. Sprangers and Schwartz [4] proposed a theoretical model for change in the meaning of self-evaluations, which they called 'response shift,' a term coined by Howard et al. [5]. Sprangers and Schwartz distinguish three types of response shift: recalibration refers to a change in respondents' internal criteria with which they assess the construct of interest; reprioritization refers to a change in respondents' values regarding the relative 
importance of subdomains; and reconceptualization refers to a change in the meaning of the target construct. Although various refined definitions and theoretical models have subsequently been proposed [6-8], they all share this working definition of response shift. While response shift can often be considered a beneficial treatment or time effect, its effect may lead to an over- or under-estimation of intervention effects, hindering the interpretation of change in HRQL outcomes. It is, thus, important to detect and take into account possible response shift effects.

Structural equation modeling (SEM) is currently the most widely used statistical approach for the investigation of response shift [9] and has been applied to examine response shift in various patient populations, disease types, and PRO measures. However, application of SEM is relatively complex as it includes many steps that require several decisions regarding, for example, the number and types of response shift to consider. When one is not aware of these different decisions and their consequences, there is a risk of using the SEM method inappropriately. Moreover, there is a lack of non-technical explanations of SEM for response shift detection; the original paper by Oort [10] is difficult to follow for non-statisticians as it contains many technical specifications, makes an unnecessary distinction between two types of recalibration, and distinguishes several other types of change that are not directly relevant for response shift investigation. The aim of the current paper is therefore to provide an accessible description of the SEM method and its associated sequential decision-making practices, in order to stimulate valid applications and interpretations of SEM for the investigation of response shift and change in HRQL outcomes. Specifically, we describe the operationalization and interpretation of change with SEM addressing only those parameters of interest for the detection of recalibration, reprioritization, and reconceptualization response shift and 'true' change in the target construct (i.e., change in the target construct while taking into account response shift), and discuss practical considerations in the application of the SEM approach. In doing so, we provide guidelines and recommendations for the investigation and interpretation of change and response shift.

Our paper is targeted at researchers who are interested in applying SEM for response shift detection and are familiar with latent variable modeling (e.g., see [11]). Note that SEM can be used to investigate response shift from both a conceptual and a measurement perspective (see [9] for formal definitions of both perspectives). In the current paper, we address response shift investigation from the measurement perspective, where response shift is defined as a change in the relation between the underlying (latent) target construct (e.g., HRQL) and the observed questionnaire responses. To explain the SEM method, enhance its accessibility, and facilitate the interpretation of its results, we use an example of HRQL measurement over time. However, we would like to emphasize that response shift can occur - and be investigated - in any PRO measure (PROM).

\section{Operationalization and interpretation of change and response shift}

Suppose cancer patients are administered a HRQL questionnaire prior to and at the end of chemotherapy. We have their scores on nine different items from a HRQL questionnaire that measures physical (i.e., 'nausea,' 'pain,' and 'fatigue'), mental ('anxiety,' 'sadness,' and 'happiness') and social ('family relations,' 'friendships,' and 'work relations') aspects of health. SEM is a statistical technique that can be used to model relationships between observed responses (e.g., patients' scores on the nine items of the HRQL questionnaire) to be reflective of one or more unobserved latent variables or common factors (e.g., the three domains of the HRQL construct that the items aim to measure) (see Fig. 1). Within the SEM framework, the variances and covariances ( $\boldsymbol{\Sigma}$, 'Sigma') and means ( $\boldsymbol{\mu}$, 'mu') of the observed variables $(X)$ are given by

$\operatorname{Cov}\left(X, X^{\prime}\right)=\boldsymbol{\Sigma}=\boldsymbol{\Lambda} \boldsymbol{\Phi} \Lambda^{\prime}+\boldsymbol{\Theta}$,

and

$\operatorname{Mean}(X)=\boldsymbol{\mu}=\boldsymbol{\tau}+\boldsymbol{\Lambda} \boldsymbol{\kappa}$,

where $\boldsymbol{\Lambda}$ ('Lambda') is a matrix of common factor loadings that describes the relationships between the observed variables and underlying common factors (e.g., the relationships

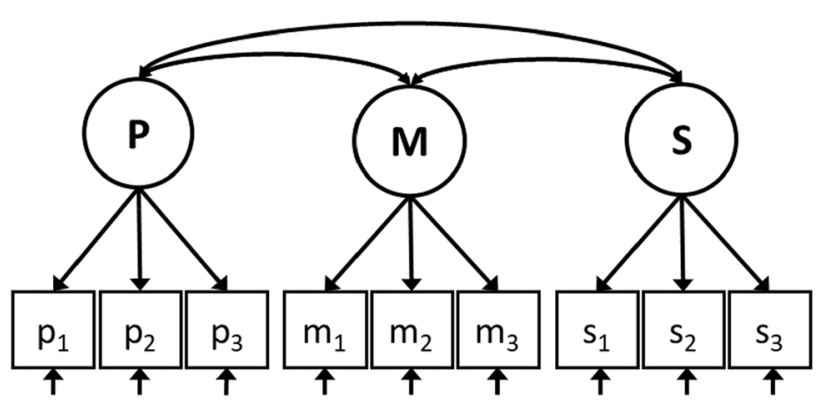

Fig. 1 A SEM model for physical (P), mental (M), and social (S) health. The squares at the bottom represent nine observed indicators, where $p_{1}$ to $p_{3}$ refer to the three measures of physical health (i.e., 'nausea,' 'pain,' and 'fatigue'), $m_{1}$ to $m_{3}$ refer to the three measures of mental health (i.e., 'anxiety,' 'sadness,' and 'happiness') and $s_{1}$ to $s_{3}$ refer to the three measures of social health (i.e., 'family relations,' 'friendships,' and 'work relations'). The solid single-headed arrows at the bottom of the squares represent the residual factors of each indicator variable. The circles at the top represent the underlying latent variables that measure everything that the indicators that load on that factor have in common [i.e., a physical (P), mental (M), and social (S) domain of HRQL]. Each arrow from a latent variable to an observed indicator represents a factor loading. The solid double-headed arrows between the latent variables represent common factor covariances 
between the underlying common factor mental health and the three associated item scores are specified by three common factor loadings), $\boldsymbol{\Phi}$ ('Phi') is a matrix of common factor variances and covariances that describes the relationships between the underlying factors (e.g., the relations between physical, mental, and social health), $\boldsymbol{\Theta}$ ('Theta') is a matrix of residual variances and covariances that cannot be explained by the underlying common factors (e.g., the variances of the nine observed item scores that cannot be explained by the three underlying common factors), $\boldsymbol{\tau}$ ('tau') is a vector of intercepts (e.g., one intercept value for each of the nine item scores), and $\boldsymbol{\kappa}$ ('kappa') is a vector of common factor means (e.g., the means of the underlying common factors physical, mental, and social health). The full matrices of the SEM model for the example from Fig. 1 are provided in Online Appendix A.

\section{Assessment of different types of change}

SEM can be applied to data from multiple measurement occasions to assess change (see Fig. 2). Specifically, the SEM method for the investigation of different types of changes in HRQL outcomes [10] uses change in the pattern of factor loadings, values of factor loadings, and intercepts to operationalize reconceptualization, reprioritization, and recalibration, respectively. In the presence of response shift, the meaning of the construct is not consistent across time. In other words, a comparison of the indicators for which response shift has been detected is compromised, as change in the observed indicators does not (only) reflect change in the underlying variables. A decomposition of change can be used to investigate the impact of response shift on change in the observed indicators [12]. Moreover, SEM enables the investigation of change in the underlying latent variables, while taking into account possible response shifts. Changes in the common factor means across occasions are indicative of 'true' change in the construct of interest. Table 1 provides an overview of the four steps of the SEM approach as proposed by Oort [10], including examples of the interpretation of response shift.

\section{Added value of the SEM approach}

There are three main advantages of the SEM approach to investigate change in HRQL outcomes. First, it allows for an operationalization of different types of response shift. Second, it can account for the different types of response shift. Third, the flexibility of the SEM framework enables the inclusion of multiple measurements (e.g., analyze more extensive follow-up designs; see [13]), multiple groups (e.g., compare different patient groups based on disease, treatment, or patient characteristics; see [14]), multidimensional scales (e.g., include multiple HRQL domains, or other latent variables, simultaneously; see [15]), or variables with different measurement levels (e.g., continuous subscale scores and categorical item scores; see [16]), and exogenous variables that possibly explain response shift. For an interpretation of the impact of response shift on the assessment of change, it is also possible to calculate SEM-based effect-size indices [12].

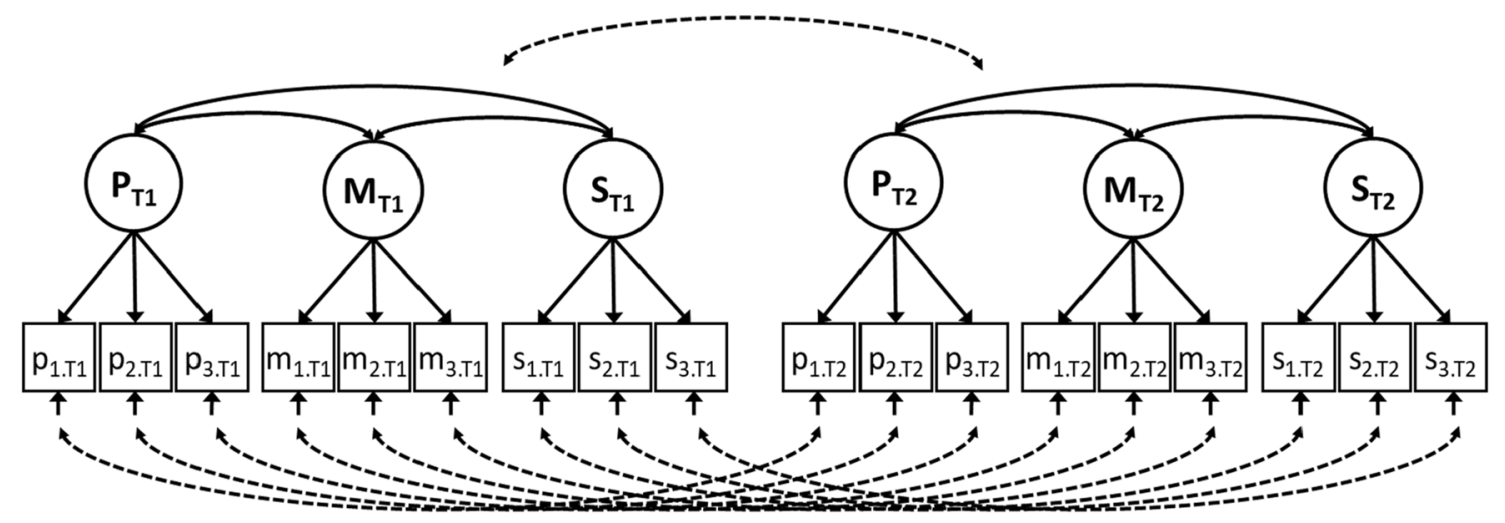

Fig. 2 A longitudinal SEM model for the investigation of change and response shift in physical $(\mathrm{P})$, mental $(\mathrm{M})$, and social $(\mathrm{S})$ health. This is the longitudinal SEM model of the same HRQL measurement as depicted in Fig. 1. The squares at the bottom represent the observed indicators, measuring physical $\left(p_{1}\right.$ to $\left.p_{3}\right)$, mental $\left(m_{1}\right.$ to $\left.m_{3}\right)$, and social $\left(s_{1}\right.$ to $\left.s_{3}\right)$ aspects of health (see Fig. 1) at two occasions (T1 and $\mathrm{T} 2$ ). The solid single-headed arrows at the bottom of the squares represent the residual factors of each indicator variable. The dotted double-headed arrows represent the longitudinal relations between the residual factors, where only the residual factors of the same indi- cator are allowed to correlate. The circles at the top represent the underlying latent variables that measure everything that the indicators that load on that factor have in common [i.e., a physical (P), mental (M), and social (S) domains of HRQL, both at T1 and T2]. Each arrow from a latent variable to an observed indicator represents a factor loading. The solid double-headed arrows between the latent variables represent common factor covariances. The dotted doubleheaded arrow represents the (nine) longitudinal correlations between the common factors 


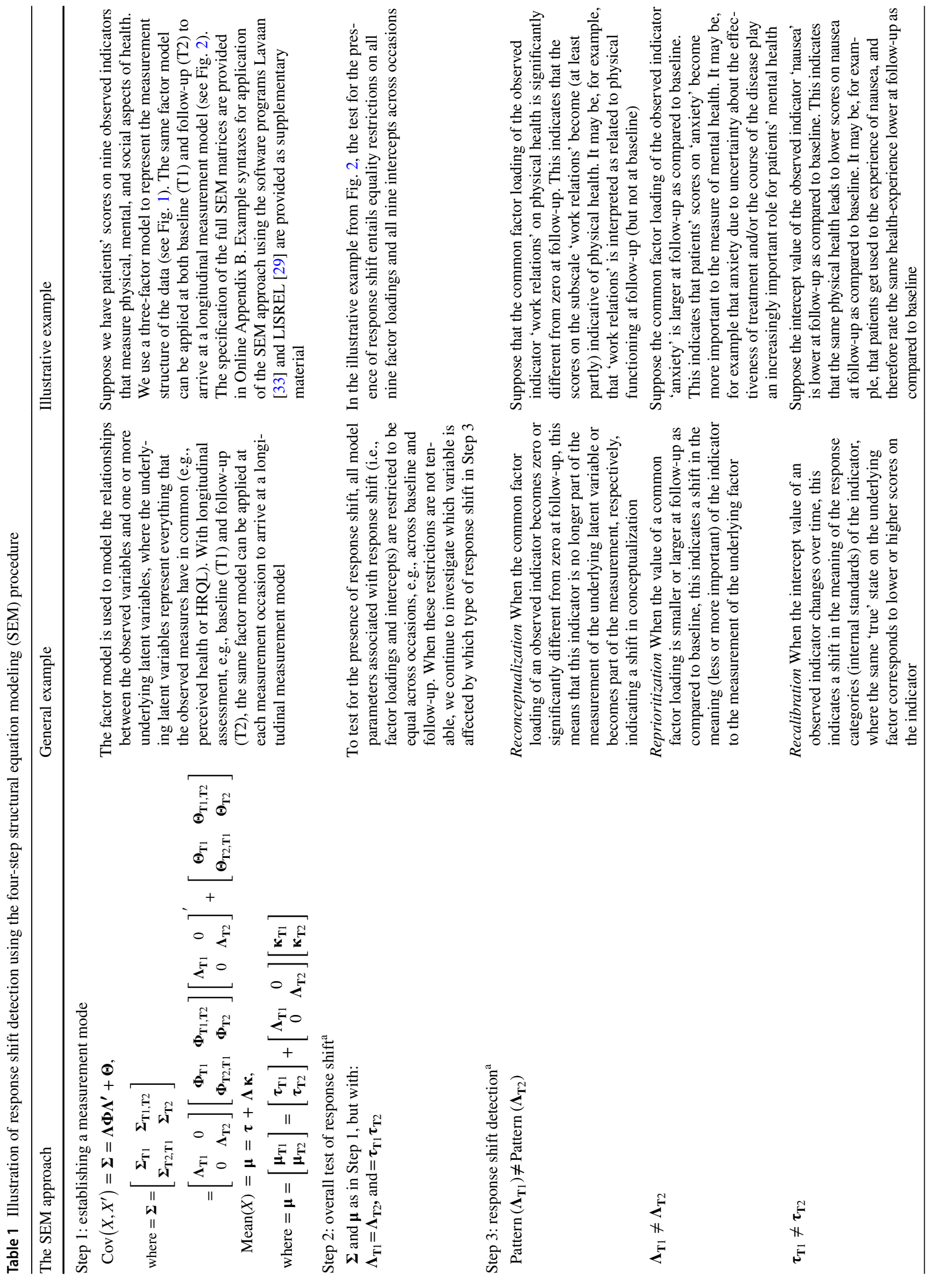




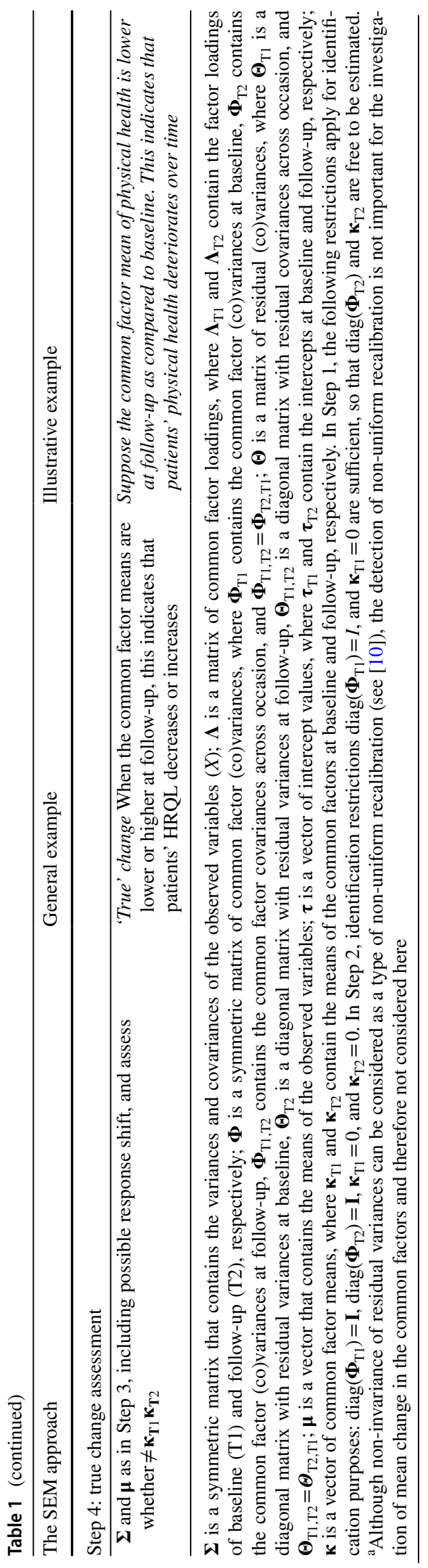

\section{Practical considerations in application of the SEM approach}

As with any method, the validity of the SEM method depends on certain methodological and conceptual assumptions. General discussions about the underlying assumptions of SEM (e.g., [11, 17]), and the measurement of latent variables (e.g., $[18,19])$ can be found elsewhere. Here, we focus on practical issues that are specifically important for valid application and interpretation of the SEM approach for detecting response shift in HRQL outcomes. Table 2 lists these issues and connects them to the four different steps in the SEM procedure.

\section{Know your measures: establishing an appropriate measurement model}

The measurement model specifies the relations between the observed variables and underlying latent factor(s) and thus defines the construct that we intend to measure. With longitudinal data, the measurement model includes the specification of the measurement structure at each measurement occasion and also referred to as the longitudinal measurement model (LMM). To arrive at the LMM (i.e., step 1 of the SEM approach), one can establish an appropriate measurement model for each measurement occasion separately and combine all separate measurement models into a single LMM (cf. [20]). Or, alternatively, one can combine all measurement occasions into a single longitudinal measurement model and establish an appropriate LMM for all measurement occasions simultaneously (cf. [21]). The only requirements of the specified LMM are that the measurement structure is largely the same (e.g., the same number of underlying common factors) across time, and that it has interpretable common factors. In practice of response shift detection, however, differences in the measurement structure are indicative of reconceptualization response shift. Therefore, the measurement structure is often specified to be the same at each occasion. For example, the LMM of our illustrative example of HRQL is specified by using the three-factor model from Fig. 1 at both baseline and follow-up (see Fig. 2).

An appropriate starting point for the specification of a measurement model can be based on the structure of the questionnaire, results from previous research, substantive considerations about the content of the observed measures, exploratory factor analyses, or-more likely-a combination of these approaches. For example, when a HRQL questionnaire is developed based on the idea that the items reflect social, mental, and physical aspects of health, then the measurement model could be specified 


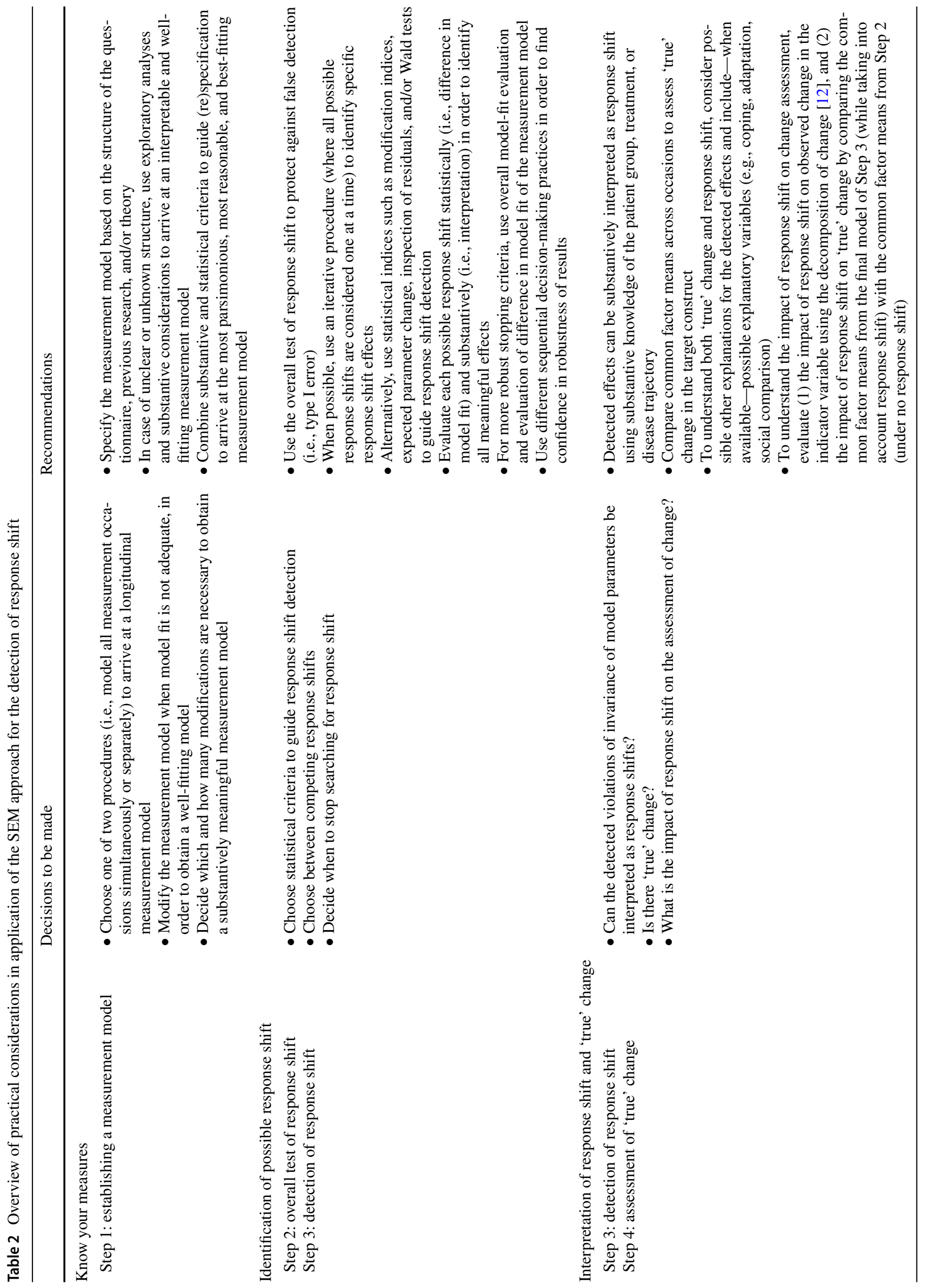


as a three-factor model, where all items that measure the same domain load on the associated common factor (such as in Fig. 1). However, specification of the measurement model can become more complicated in situations where the dimensional structure of a questionnaire is unclear, or where (items of) different questionnaires are combined (cf. [14]). Moreover, it is often necessary to modify the initially specified measurement model to obtain a wellfitting model. A well-fitting measurement model is necessary, as the measurement model is the baseline model against which all further models (that are used to test for the presence of response shift) will be compared. Thus, the measurement model represents the most parsimonious, the most reasonable or defendable, and the best-fitting model to the data [22].

To evaluate whether the model fit of the measurement model is appropriate (e.g., assessment of overall model fit) and to guide model specification, when the initial model fit is suboptimal or inadequate (e.g., using differences in model fit), one can use statistical criteria. However, evaluation of statistical criteria for (differences in) model fit is complicated by the fact that there exist many different fit indices, with different decision rules that may be more or less appropriate depending on the context of the study. An overview of the most important fit measures and their (dis) advantages are provided in Table 3. As a general recommendation, the researcher could inspect and report several fit indices but should be aware that choice of the specific fit index might depend on the specifics of the data (e.g., sample size), complexity of the model, and/or the hypothesis that is being tested. Detailed discussions on the use of different SEM-based fit indices are provided elsewhere (e.g., see [23-25]).

Making decisions in model (re-)specification also require substantive considerations (i.e., does a model make sense?). For example, statistical indices may indicate that the largest improvement in fit can be achieved by freeing a factor loading of a physical functioning item on a common factor that measures mental health; such a model specification may not make sense substantively. On the other hand, freeing a residual covariance between indicators that share the same item format may be sensible cf. [26] even though it will not lead to a large improvement in model fit or to a change in interpretation of the common factors. In order to find a substantively reasonable measurement model, it is at least equally — and possibly even more-important to rely on substantive knowledge as on statistical criteria.

\section{Identification of possible response shift}

The mere presence of response shift is evaluated by testing whether the equality restrictions on all model parameters associated with response shift are tenable (i.e., Step 2 of the SEM procedure), representing an 'omnibus test' for the presence of response shift. This procedure has also been advocated by others [27] and has been shown to protect against false positives [28]. However, if there is evidence of the presence of response shift, how does one then accurately locate which observed variable is affected by which type of response shift?

The search for response shift (i.e., step 3 of the SEM approach) requires exploratory model fitting or re-specification, which is referred to as the 'specification search.' The specification search can be guided using statistical criteria, such as modification indices, expected parameter changes, Wald tests, inspection of residuals, or differences in model fit [29]. In order to correctly identify the change in model parameters, it has been recommended to use an iterative procedure [30], where all model parameters associated with response shift are freed one at a time, and the freely estimated parameter that shows the largest improvement in model fit is incorporated in the model. However, it may be that two different model modifications lead to equivalent improvement in model fit. A decision on which model modification to prioritize can, therefore, not be based on statistical criteria alone. Given the dependence of sequential model re-specification, freeing one model parameter may render freeing the other model parameter unnecessary, i.e., a change to the model can affect other parts of the model too. It may therefore be possible that alternative series of model re-specifications lead to different results. For example, in the search for response shift in our illustrative example of HRQL, it may be that freeing the intercept value of either 'family relations' or 'friendships' (both indicators of social health; see Fig. 1) would lead to an equivalent improvement in model fit, but that freeing one would render freeing the other intercept unnecessary. One thus needs other-substantive-reasons to decide on which response shift effect to include in the model. It may be, for example that recalibration of 'family relations' is much more plausible given the type of catalyst (e.g., type of disease or treatment) or prevalence of married patients/marital status in the study population.

Instead of strictly adhering to a procedure where only the modification that leads to the largest model-fit improvement is considered, it may be important to follow different sequences in model re-specification-i.e., choose different modifications that lead to different but more-or-less equivalent model-fit improvement- to investigate whether and to what extent these different sequential decisions lead to different results. This will allow the researcher to see whether detection of response shift is dependent on sequential decision-making practices and to choose among possible differences in these sequences based on a combination of both statistical and substantive considerations. It is this repeated back-and-forth specification search in which one can find 


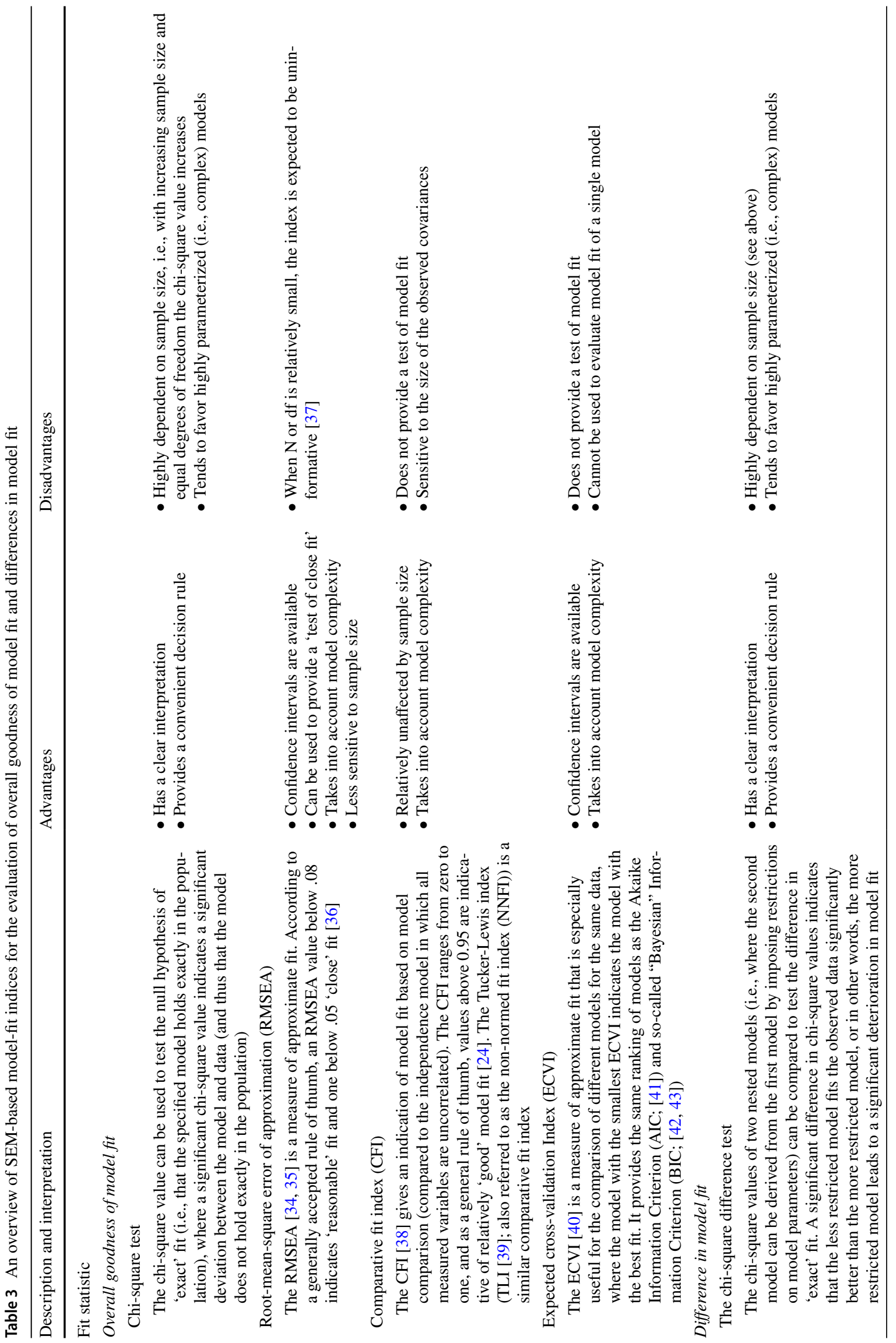


confidence in the robustness of results or, alternatively, find that a confident conclusion about the number and types of response shift is not warranted. Clearly, these sequential decision-making practices thus require subjective judgment, and different researchers may make different decisions. This is a necessary consequence of ensuring interpretability of findings. For example, it may be that in different sequences of response shift investigation for our illustrative example of HRQL, the difference in intercepts of 'family relations' re-occurs frequently, while the difference in intercepts of 'friendships' only occurs sporadically. Such a pattern of results may help to decide between different modifications that lead to similar improvements in model fit.

The specification search for possible response shift effects also requires a decision on when to stop searching. The aim of the specification search is to identify all possible response shift effects (i.e., identify all true positives). Meanwhile, however, one wants to prevent the identification of trivial differences in model parameters across time as being of substantive interest (i.e., identification of false positives, or type 1 errors). In addition to the improvement in model fit for freeing individual parameters, one can rely on the difference in model fit between the measurement model and the model that includes all identified response shift effects. When the overall difference in fit between these models is not significant, this may be taken as an indication that freeing additional model parameters is no longer necessary. Also, one can use the overall model fit of the model to judge whether the model that includes response shift is tenable. These model-fit evaluations may provide more robust stopping criteria. However, it has also been argued that in order to adequately identify all response shift effects, it may be necessary to continue the specification search, even when the established model already shows adequate model fit [31]. Therefore, model-fit criteria should be used in combination with substantive criteria with regard to the (possible) response shifts. For example, it may be that freeing an additional model parameter will lead to a small, non-significant improvement in model fit, but that the associated response shift has a clear interpretation. For example, when in our illustrative example of HRQL, there is an a-priori hypothesis about the occurrence of reprioritization response shift of 'nausea' (see Table 1), it may be informative to report on a small but non-significant effect. As a researcher, one has to find a balance between the goodness of fit and the interpretability of the model. Again, subjective judgment is needed to ensure meaningfulness of the results.

\section{Interpretation of detected response shift and 'true' change}

With SEM, we do not look at response shifts directly, but at the effects, these response shifts have on the measurement 
of change in HRQL. This allows us to describe what occurs (i.e., patterns of different types of change), but it does not imply that we also know how it occurs (i.e., what the causes are of the identified response shift). For the substantive interpretation of change, it is therefore important to provide an interpretation and possible explanation of detected response shift. For example, imagine that in our illustrative example, recalibration was detected in the indicator 'pain' of physical health, where patients showed a larger decrease in pain as compared to the other indicators of physical health. A possible explanation for this result may be that patients adapted to the experience of pain and therefore rated their pain to be lower at follow-up, even though their actual experience of pain did not change (or changed to a lesser degree), i.e., recalibration response shift (see Table 1). It may also be that patients received treatment or medication that reduced their experienced level of pain. However, one could argue that only the first interpretation coincides with what Sprangers and Schwartz [4] describe as recalibration response shift. The SEM approach for the detection of response shift does not make such substantive distinctions. Therefore, substantive interpretation of detected response shift is of paramount importance; it is needed both to clarify what is taken as evidence of response shift and to exclude, or make less likely, alternative explanations.

The interpretation of detected response shifts can be based on substantive knowledge of the patient group, the treatment, or disease trajectory. In addition, it is possible to include operationalizations of potential explanations of response shift in the SEM model. If measures of antecedents (e.g., sociodemographic or personality characteristics) or mechanisms (e.g., coping strategies, social comparison) are available, they can be incorporated in the model as possible explanatory variables for response shift effects cf. [32]. For example, in order to investigate the role of appraisal processes (following [7]) for the detected recalibration response shift of pain as described above, one could include a direct measure of appraisal in the model and investigate the effect of appraisal on the (change in) scores of the indicator 'pain.' Such investigations will help to substantiate whether and how the detected response shifts are influenced by individuals' cognitive changes in standards, values, or conceptualizations. As such, substantive interpretation and explanation of response shift are necessary to understand both the mechanisms of response shift, and how it affects change in the construct that we intend to measure (i.e., HRQL), which in turn will help to better understand patients' perceived health trajectories.

Finally, the (clinical) relevance of occurrences of response shift can be evaluated by calculating the impact of response shift on the assessment of change. First, the decomposition of change [12] can be used to interpret the impact of response shift on change in the observed variables (e.g., change in item scores). The decomposition entails that observed change is decomposed into so-called 'true' change (i.e., change due to change in the underlying target construct) and change due to response shift. Second, the impact of response shift on 'true' change in the underlying target construct (e.g., HRQL) can be evaluated by comparing estimates of change before and after taking into account response shifts. SEM-based effect-size indices can help to interpret the magnitude of the impact on change assessment [12]. This is important because substantial and interpretable response shifts do not always exert a considerable impact on 'true' change. For example, it may be that the detected recalibration response shift in the indictor 'pain' is statistically significant, interpretable (see above), and has substantial impact on the observed change in pain. At the same time, it may be that 'true' change in physical health is not influenced by the detected response shift. Then, the detected recalibration response shift has no impact on the interpretation of change in HRQL. Still, the occurrence (and investigation) of response shift is insightful because it shows how change in the target construct is (differentially) related to change in the observed measures. Both types of information regarding the impact of response shift on change assessment can thus be used to better interpret the findings from response shift investigations.

\section{Conclusion}

In the current paper, we discuss practical issues that are important for researchers who want to apply SEM for the assessment of change and detection of response shift. We provide general recommendations that can be used for all applications, while acknowledging that decisions are made on a case-by-case basis and require the substantive issues at stake. We wish to emphasize the importance of taking into account substantive considerations in addition to statistical information to guide the sequential decision-making practices. These decisions require subjective judgment and are needed for any statistical modeling procedure to ensure interpretability of findings. Moreover, for a meaningful interpretation of change, it is important to try to substantiate the linkage between detected response shift and patients' perceived health trajectories, e.g., by using substantive knowledge or direct measures of possible explanatory variables. With the recommendations provided in this paper, we aim to stimulate the appropriate application and interpretation of SEM for the investigation of response shift and assessment of change in PROs and thus improve the scientific stringency of the field. As sound statistical techniques can contribute to a better understanding of patients' perceived health trajectories, this will ultimately improve the 
evaluation and interpretation of the effectiveness of healthcare interventions and thus improve the quality of patients' lives.

Acknowledgements Parts of this manuscript are based on Chapters 1 (Introduction) and 9 (General discussion) of the doctoral dissertation of M.G.E. Verdam [45] that has been published under the Creative Commons License.

\section{Compliance with ethical standards}

Conflict of interest The authors declare that they have no conflict of interest.

Open Access This article is licensed under a Creative Commons Attribution 4.0 International License, which permits use, sharing, adaptation, distribution and reproduction in any medium or format, as long as you give appropriate credit to the original author(s) and the source, provide a link to the Creative Commons licence, and indicate if changes were made. The images or other third party material in this article are included in the article's Creative Commons licence, unless indicated otherwise in a credit line to the material. If material is not included in the article's Creative Commons licence and your intended use is not permitted by statutory regulation or exceeds the permitted use, you will need to obtain permission directly from the copyright holder. To view a copy of this licence, visit http://creativecommons.org/licenses/by/4.0/.

\section{References}

1. Fayers, P., \& Machin, D. (2016). Quality of life. The assessment, analysis and reporting of patient-reported outcomes (3rd ed.). Wiley: West Sussex.

2. Huber, M., Knottnerus, J. A., Green, L., van der Harst, H., Jadad, A. R., et al. (2011). How should we define health?. British Medical Journal, 343, d4163.

3. Ferrans, C. E. (2007). Differences in what quality-of-life instruments measure. Journal of the National Cancer Institute Monographs, 37, 22-26.

4. Sprangers, M. A. G., \& Schwartz, C. E. (1999). Integrating response shift into health-related quality of life research: A theoretical model. Social Science and Medicine, 48, 1507-1515.

5. Howard, G. S., Ralph, K. M., Gulanick, N. A., Maxwell, S. E., Nance, S. W., \& Gerber, S. K. (1979). Internal invalidity in pretest-posttest self-report evaluations and reevaluation of retrospective pretests. Applied Psychological Measurement, 3, 1-23.

6. Rapkin, B. D., \& Schwartz, C. E. (2004). Toward a theoretical model of quality-of-life appraisal: Implications of findings from studies of response shift. Health and Quality of Life Outcomes, 2(1), 14.

7. Rapkin, B. D., \& Schwartz, C. E. (2019). Advancing quality-of life research by deepening our understanding of response shift: A unifying theory of appraisal. Quality of Life Research, 28(10), 2623-2630.

8. Oort, F. J., Visser, M. R. M., \& Sprangers, M. A. G. (2009). Formal definitions of measurement bias and explanation bias clarify measurement and conceptual perspectives on response shift. Journal of Clinical Epidemiology, 62(11), 1126-1137.

9. Sajobi, T. T., Brahmbatt, R., Lix, L. M., Zumbo, B. D., \& Sawatzky, R. (2018). Scoping review of response shift methods: Current reporting practices and recommendations. Quality of Life Research, 27, 1133-1146.
10. Oort, F. J. (2005). Using structural equation modeling to detect response shift and true change. Quality of Life Research, 14, 587-598.

11. Bollen, K. A. (1989). Structural equations with latent variables. New York: Wiley.

12. Verdam, M. G. E., Oort, F. J., \& Sprangers, M. A. G. (2017). Structural equation modeling-based effect-size indices were used to evaluate and interpret the impact of response shift effects. Journal of Clinical Epidemiology, 85, 37-44.

13. Verdam, M. G. E., \& Oort, F. J. (2019). The analysis of multivariate longitudinal data: An instructive application of the longitudinal three-mode model. Multivariate Behavioral Research, 54(4), 457-474.

14. Verdam, M. G. E., Oort, F. J., van der Linden, Y. M., \& Sprangers, M. A. G. (2015). Taking into account the impact of attrition on the assessment of response shift and true change: A multigroup structural equation modeling approach. Quality of Life Research, 24, 541-551.

15. Verdam, M. G. E., Oort, F. J., \& Sprangers, M. A. G. (2017). Using structural equation modeling to detect item bias: Comparison with other item bias detection methods. Quality of Life Research, 26, 1439-1450.

16. Verdam, M. G. E., Oort, F. J., \& Sprangers, M. A. G. (2016). Using structural equation modeling to detect response shifts and true change in discrete variables: An application to the items of the SF-36. Quality of Life Research, 25, 1361-1383.

17. Bentler, P. M., \& Chou, C.-P. (1987). Practical issues in structural modeling. Sociological Methods and Research, 16, 78-117.

18. Borsboom, D., Mellenberg, G. J., \& van Heerden, J. (2003). The theoretical status of latent variables. Psychological Review, 110, 203-219.

19. Cohen, P., Cohen, J., Teresi, J., Marchi, M., \& Velez, C. N. (1990). Problems in the measurement of latent variables in structural equation causal models. Applied Psychological Measurement, 14, 183-196.

20. Ahmed, S., Bourbeau, J., Maltais, F., \& Mansour, A. (2009). The Oort structural equation modeling approach detected a response shift after a COPD self-management program not detected by the Schmitt technique. Journal of Clinical Epidemiology, 62, 1165-1172.

21. Nolte, S., Elsworth, G. R., Sinclair, A. J., \& Osborne, R. H. (2009). Tests of measurement invariance failed to support the application of the "then-test." Journal of Clinical Epidemiology, 62, 1173-1180.

22. Byrne, B. M., Shavelson, R. J., \& Muthén, B. (1989). Testing for the equivalence of factor covariance and mean structures: The issue of partial measurement invariance. Psychological Bulletin, 105, 456-466.

23. Schermelleh-Engel, K., Moosbrugger, H., \& Müller, H. (2003). Evaluating the fit of structural equation models: Tests of significance and descriptive goodness-of-fit measures. Methods of Psychological Research Online, 8, 23-74.

24. Hu, L., \& Bentler, P. M. (1999). Cutoff criteria for fit indexes in covariance structure analysis: Conventional criteria versus new alternatives. Structural Equation Modeling, 6, 1-55.

25. Marsh, H. W., Hau, K.-T., \& Wen, Z. (2004). In search of golden rules: Comment on hypothesistesting approaches to setting cutoff values for fit indexes and dangers in overgeneralizing $\mathrm{Hu}$ and Bentler's (1999) findings. Structural Equation Modeling, 11, 320-341.

26. Oort, F. J., Visser, M. R. M., \& Sprangers, M. A. G. (2005). An application of structural equation modeling to detect response shifts and true change in quality of life data from cancer patients undergoing invasive surgery. Quality of Life Research, 14, 599-609. 
27. Millsap, R. E. (2010). Testing measurement invariance using item response theory in longitudinal data: An introduction. Child Development Perspectives, 4, 5-9.

28. Vanier, A., Sébille, V., Blanchin, M., Guilleux, A., \& Hardouin, J.-B. (2015). Overall performance of Oort's procedure for response shift detection at item level: A pilot simulation study. Quality of Life Research, 24, 1799-1807.

29. Jöreskog, K. G., \& Sorbom, D. (1996). LISREL 8 users'guide (2nd ed.). Chcago, IL: Scientific Software International Inc.

30. Cheung, G. W., \& Rensvold, R. B. (1999). Testing factorial invariance across groups: A reconceptualization and proposed new method. Journal of Management, 25, 1-27.

31. MacCallum, R. (1986). Specification searches in covariance structure modeling. Psychological Bulletin, 100, 107-120.

32. King-Kallimanis, B. L., Oort, F. J., Visser, M. R. M., \& Sprangers, M. A. G. (2009). Structural equation modeling of health-related quality-of-life data illustrates the measurement and conceptual perspectives on response shift. Journal of Clinical Epidemiology, 62, 1157-1164.

33. Rosseel, Y. (2012). Lavaan: An R package for structural equation modeling. Journal of Statistical Software, 48, 1-36.

34. Steiger, J. H., \& Lind, J. C. (1980). Statistically based tests for the number of common factors. Iowa City, IA: Paper presented at the annual meeting of the Psychometric Society.

35. Steiger, J. H. (1990). Structural model evaluation and modification: An interval estimation approach. Multivariate Behavioral Research, 25, 173-180.

36. Browne, M. W., \& Cudeck, R. (1992). Alternative ways of assessing model fit. Sociological Methods Research, 21, 230-258.
37. Kenny, D. A., Kaniskan, B., \& McCoach, D. B. (2015). The performance of RMSEA in models with small degrees of freedom. Sociological Methods \& Research, 44(3), 486-507.

38. Bentler, P. M. (1990). Comparative fit indexes in structural models. Psychological Bulletin, 107, 238-246.

39. Tucker, L. R., \& Lewis, C. (1973). A reliability coefficient for maximum likelihood factor analysis. Psychometrika, 38, 1-10.

40. Browne, M. W., \& Cudeck, R. (1989). Single sample cross-validation indices for covariance structures. Multivariate Behavioral Research, 24, 445-455.

41. Akaike, H. (1987). Factor analysis and AIC. Psychometrika, 52, 317-332.

42. Raftery, A. E. (1986). Choosing models for cross-classification. American Sociological Review, 51(1), 145-146.

43. Raftery, A. E. (1995). Bayesian model selection in social research. Sociological Methodology, 25, 111-163.

44. Cheung, G. W., \& Rensvold, R. B. (2002). Evaluating goodnessof-fit indexes for testing measurement invariance. Structural Equation Modeling, 9, 233-255.

45. Verdam, M. G. E. (2017). Using structural equation modeling to investigate change in health-related quality of life. Amsterdam: University of Amsterdam. [Unpublished doctoral dissertation].

Publisher's Note Springer Nature remains neutral with regard to jurisdictional claims in published maps and institutional affiliations. 